\title{
A family with upper limb malformations and dyspnoea - the Holt-Oram syndrome
}

Nikesh Raj Shrestha ${ }^{a}$, Stéphane Cook ${ }^{b}$

a B. P. Koirala Institute of Health Sciences, Dharan, Koshi, Nepal

b University \& Hospital Fribourg, Switzerland

\section{Case report}

A 16-year-old male presented at our outpatient clinic with palpitations and worsening dyspnoea during the preceding five days. He had been complaining of dyspnoea since he was eight and had a history of recurrent respiratory infections. Physical examination revealed small upper limb, hands with absent or triphalangeal thumbs, hypoplasia of pectoralis major muscle and

pectus excavatum (fig. 1 and 2). Cardiac auscultation demonstrated grade 3 midsystolic murmur over pulmonic area (due to increased pulmonic blood flow) with widely split and fixed S2. Sinus bradycardia, firstdegree atrio-ventricular block and right bundle branch block were present. Transthoracic echocardiography revealed ostium secundum atrial septal defect (ASD) with dilated right cavities (fig. 3). His mother and younger brother accompanied him and possessed simi-

Figure 1

The patients hands with absent or triphalangeal thumbs.

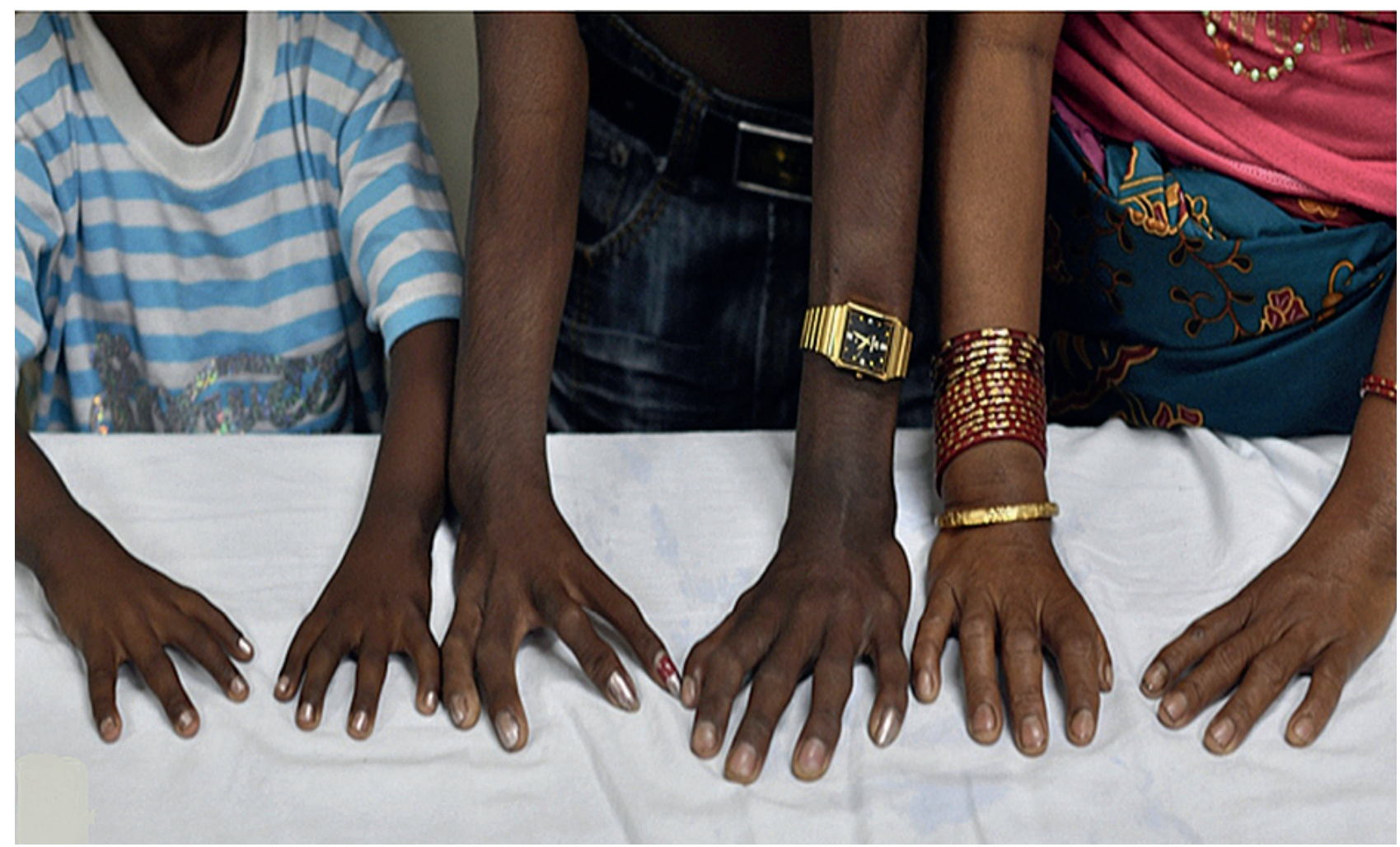


lar bone anomalies. Transthoracic echocardiography was therefore also performed in these relatives and also revealed ostium secundum ASDs.

Association of abnormalities in the upper limb together with presence of ostium secundum ASD is pathognomonic of Holt-Oram syndrome (HOS). HOS was initially described in 1960 by M. Holt and S. Oram

\section{Figure 2}

Hypoplasia of pectoralis major muscle and pectus excavatum.

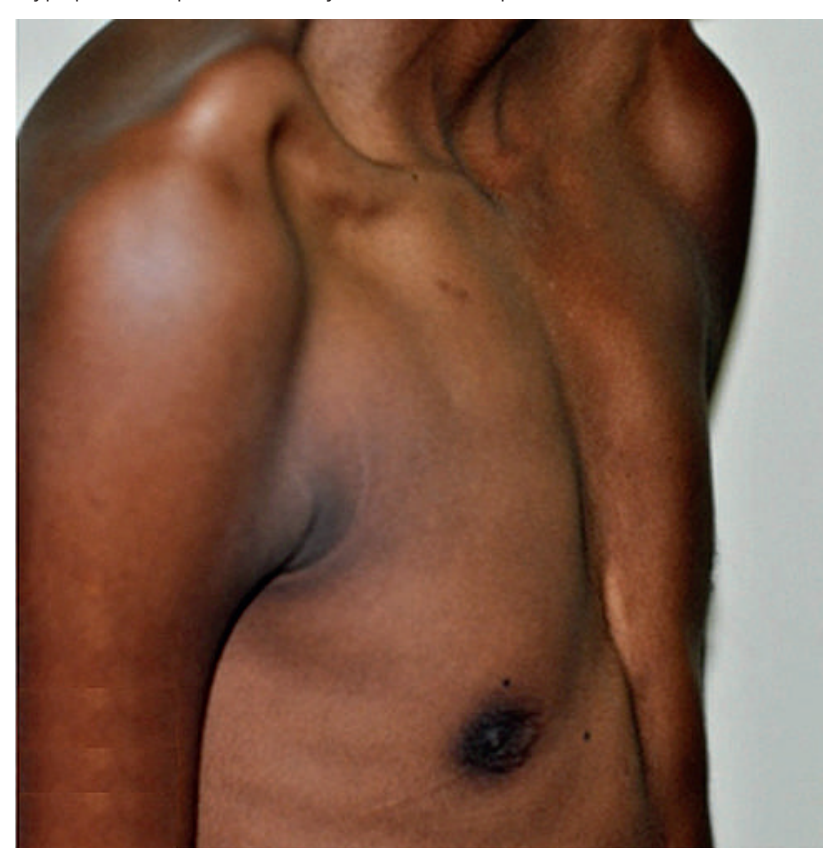

and is an autosomal dominant syndrome in which mutations in the TBX5 gene in the long arm of chromosome 12 accounted for various skeletal and cardiovascular abnormalities. However, we were not able to perform the mutation analysis in these patients as it is currently not available in Nepal.

\section{Figure 3}

Transthoracic echocardiography revealed ostium secundum atrial septal defect (ASD) with dilated right cavities.

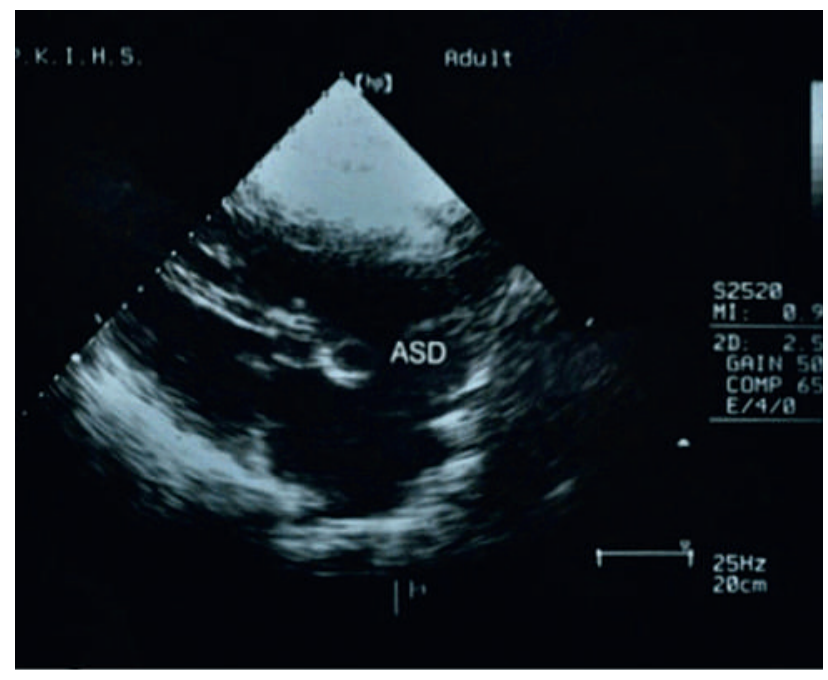

